\title{
Questioning the validity of non-extensive thermodynamics for classical Hamiltonian systems
}

\author{
James F. Lutsko ${ }^{1(a)}$ And JeAn Pierre Boon ${ }^{1(b)}$ \\ 1 Center for Nonlinear Phenomena and Complex Systems CP 231 \\ Université Libre de Bruxelles, 1050 Brussels, Belgium
}

PACS 05.20.Jj - Statistical mechanics of classical fluids

PACS 05.70.Ce - Thermodynamic functions

\begin{abstract}
We examine the non-extensive approach to the statistical mechanics of Hamiltonian systems with $H=T+V$ where $T$ is the classical kinetic energy. Our analysis starts from the basics of the formalism by applying the standard variational method for maximizing the entropy subject to the average energy and normalization constraints. The analytical results show (i) that the nonextensive thermodynamics formalism should be called into question to explain experimental results described by extended exponential distributions exhibiting long tails, i.e. $q$-exponentials with $q>1$, and (ii) that in the thermodynamic limit the theory is only consistent in the range $0 \leq q \leq 1$ where the distribution has finite support, thus implying that configurations with e.g. energy above some limit have zero probability, which is at variance with the physics of systems in contact with a heat reservoir. We also discuss the ( $q$-dependent) thermodynamic temperature and the generalized specific heat.
\end{abstract}

Introduction. - The observation of natural phenomena and of laboratory experiments provides a wide spectrum of experimental results showing distributions of data that deviate from exponential decay as predicted for Boltzmann behavior [1. It was the goal of nonextensive statistical mechanics developed originally by Tsallis [2] to offer a new approach to explain the non-Boltzmann behavior of non-equilibrium systems 1 . More precisely the primary motivation for non-extensive thermodynamics is as a way to understand deformed exponential distributions (such as $q$-exponentials exhibiting long tails when $q>1$ ) found empirically in many areas of physics and other scientific disciplines [5. The interest raised by this new approach has grown over the years and has produced an abundant literature [6] reflecting new theoretical developments and a considerable number of applications to subjects as diverse as defect turbulence, energy distribution in cosmic rays, earthquake magnitude value distributions and velocity distributions in micro-organism populations or distributions of financial market data. Parallel to these developments, critical analyses were presented questioning the merits of the non-extensive theory $[7$ and thereby of its applications as well. These criticisms raised questions that often gave rise to ontological conflicts [8]. Most of the criticisms are based on phenomenological analyses and thermodynamic arguments questioning the compatibility of the theory with classical statistical thermodynamics. More recently,

\footnotetext{
(a) E-mail: jlutsko@ulb.ac.be

(b) E-mail: jpboon@ulb.ac.be

${ }^{1}$ We note that other approaches exist such as super-statistics 3 and the fractional and nonlinear FokkerPlanck equations (see e.g. [4] and references therein)
} 
the non-extensive theory was also re-examined on the basis of analyses demonstrating the necessary discreteness of systems to which the theory applies [9] and the limits of validity of the non-extensive formalism for a Hamiltonian system, the $q$-ideal gas, a model system of independent quasi-particles where the interactions are incorporated in the particles properties [10,11. Here we merely adopt the viewpoint of analytical rigor to establish the validity limits of the non-extensive formalism for the general class of classical Hamiltonian systems with continuous variables and consequently of the class of physical systems to which the non-extensive interpretation applies.

In the formalism, the $q$-exponential distributions arise as the result of a variational calculation maximizing the generalized entropy, the $q$-entropy, under the constraints of normalizability of the distribution function and of a prescribed average energy [6]. The goal of the present work is to investigate the exact form of the distribution so derived for classical Hamiltonian systems from both the usual Tsallis entropy [2] and the homogeneous entropy [13. The conclusion is found to be the same in both cases: the theory is only consistent in the thermodynamic limit for $0 \leq q \leq 1$. For finite systems of $N$ particles, the upper limit is $1+a / N$ for $a \sim \mathcal{O}(1)$. This means (i) that the non-extensive thermodynamics formalism cannot be used, at least in any straightforward way, to explain phenomena for which one observes that $q$ takes a value $q>1$ (corresponding to asymptotically power-law distributions) i.e. when the distributions exhibit extended exponential forms with long tails, and (ii) that, within the validity domain, the distribution has finite support, thus implying that configurations with e.g. energy above some limit have zero probability, a strange situation for systems with typical molecular potentials which are steeply repulsive at small distances. We treat successively the case according to the development based on the Tsallis entropy $S_{q}$ and the case based on the homogeneous entropy $S_{q}^{H}$. The reason for examining the two cases is the criterion of stability against perturbations of the probability distribution function, or Lesche stability 12 : the homogeneous entropy was shown to be Lesche stable while the Tsallis entropy is not [13. We also present the results for the generalized (q-dependent) thermodynamic properties of Hamiltonian systems in both cases.

Tsallis entropy. - Non-extensive statistical mechanics is developed on the basis of three axioms: (i) the $q$-entropy for systems with continuous variables is given by 6 ]

$$
S_{q}=k_{B} \frac{1-K \int \rho^{q}(\Gamma) d \Gamma}{q-1}
$$

where $\Gamma$ is the phase space variable and $K$ must be a quantity with the dimensions of $[\Gamma]^{q-1}$, i.e. $K=\hbar^{D N(q-1)}$ ( $D$ denotes the dimension of the system and $N$ the number of particles) and the classical Boltzmann-Gibbs entropy is retrieved in the limit $q \rightarrow 1$; (ii) the distribution function $\rho(\Gamma)$ is slaved to the normalization condition

$$
1=\int \rho(\Gamma) d \Gamma
$$

(iii) the internal energy is measured as

$$
U=\int P_{q}(\Gamma) H d \Gamma=\frac{\int \rho^{q}(\Gamma) H d \Gamma}{\int \rho^{q}(\Gamma) d \Gamma},
$$

where $P_{q}(\Gamma)$ is the escort probability distribution [14 which is the actual probability measure. We consider Hamiltonian systems with $H=T+V$ where $T$ is the classical kinetic energy. The distribution function $\rho(\Gamma)$ is obtained by maximizing the $q$-entropy subject to the normalization (2) and average energy (3). Introducing the Lagrange multipliers $\bar{\alpha}$ and $\bar{\gamma}$, the variational method leads to

$$
0=\frac{k_{B} K q}{1-q} \rho^{q-1}(\Gamma)-\bar{\alpha}-q \bar{\gamma} \frac{(H-U)}{\int \rho^{q}(\Gamma) d \Gamma} \rho^{q-1}(\Gamma),
$$


which is solved to yield

$$
\rho(\Gamma)=\frac{(1-(1-q) \gamma(H-U))^{\frac{1}{1-q}}}{\int(1-(1-q) \gamma(H-U))^{\frac{1}{1-q}} d \Gamma},
$$

with $\gamma=\frac{\bar{\gamma}}{K \int \rho^{q}(\Gamma) d \Gamma}$ and where the normalization condition (2) has been used to eliminate the multiplier $\bar{\alpha}$. The other, $\gamma$, is determined from the energy constraint (3); using (5) in (3), we have

$$
0=\frac{\int(1-(1-q) \gamma(H-U))^{\frac{q}{1-q}}(H-U) d \Gamma}{\int(1-(1-q) \gamma(H-U))^{\frac{q}{1-q}} d \Gamma},
$$

which by multiplying the numerator by $(1-q) \gamma$ and adding and subtracting one gives

$$
\int(1-(1-q) \gamma(H-U))^{\frac{q}{1-q}} d \Gamma=\int(1-(1-q) \gamma(H-U))^{\frac{1}{1-q}} d \Gamma .
$$

Note that the sign of the factor $f(\Gamma) \equiv 1-(1-q) \gamma(H-U)$ is, so far, arbitrary so that we allow for the cancellation of a (possible imaginary) factor throughout these equations. However, this is only possible in the expression for the distribution (5) if the sign of $f(\Gamma)$ is independent of $\Gamma$. Given this fact, the sign can be fixed by making the substitution $f(\Gamma)=s|f(\Gamma)|$ in the energy constraint which requires that $s^{\frac{q}{1-q}}=s^{\frac{1}{1-q}}$ so that $s=1$. Since the kinetic energy is in principle unbounded, the constant sign of the argument means that the distribution may have to be restricted to some subset of phase space so that it should be written as

$$
\rho(\Gamma)=\frac{(1-(1-q) \gamma(H-U))^{\frac{1}{1-q}} \Theta(1-(1-q) \gamma(H-U))}{\int(1-(1-q) \gamma(H-U))^{\frac{1}{1-q}} \Theta(1-(1-q) \gamma(H-U)) d \Gamma},
$$

where $\Theta(x)$ is the step function which is one for $x>0$ and zero otherwise. We note that the introduction of the step function is in fact a redefinition of the variational problem in the sense that we have replaced $\rho(\Gamma)$ with $\bar{\rho}(\Gamma) \Theta(f(\Gamma))$ in Eq.(1.3) and then maximized with respect to $\bar{\rho}(\Gamma)$. If we do not do this, then there is simply no solution to the variational problem which is real and non-negative for all $\Gamma$. To see this, we note from the variational equation (4) that if $\rho(\Gamma)$ vanishes for some value of $\Gamma$ then it necessarily follows that $\bar{\alpha}=0$, which is untenable since $\bar{\alpha}$ will generally assume a non-zero value due to the normalization condition. Therefore, we can only restrict the support of the distribution by redefining the variation problem. We now turn to the determination of the possible values for $q$.

Case: $q<1$. The exponent occuring in the distribution is $\frac{1}{1-q}>0$ so that if $\gamma>0$, then the distribution must have finite support since for some sufficiently large value of $T$, the argument, $f(\Gamma)$, becomes zero, and from (5) we have

$$
\rho(\Gamma)=\frac{\exp _{q}(-\gamma(H-U))}{\int \exp _{q}(-\gamma(H-U)) d \Gamma},
$$

where $\exp _{q}(x) \equiv(1+(1-q) x)^{\frac{1}{1-q}} \Theta(1+(1-q) x)$ is the $q$-exponential function.

If $\gamma<0$, then $f(\Gamma)$ is always positive, and the distribution function never goes to zero so that there can be no finite support. This however leads to another problem since $f(\Gamma)$ is unbounded as the kinetic energy increases which, in turn, means that the integral of $f(\Gamma)$ over momenta will diverge so that the distribution cannot be normalized. We conclude that $\gamma<0$ is not allowed. We remark that it might be thought that one could introduce a limit 
on the kinetic energy, but this is not in keeping with the proposal that the nonextensive distribution be a generalization of the canonical distribution which describes open systems in contact with a reservoir.

Case: $q>1$. The exponent being then $\frac{1}{1-q}<0$, we write the distribution function as

$$
\rho(\Gamma)=\frac{(1+|1-q| \gamma(H-U))^{-\left|\frac{1}{1-q}\right|} \Theta(1+|1-q| \gamma(H-U))}{\int(1+|1-q| \gamma(H-U))^{-\left|\frac{1}{1-q}\right|} \Theta(1+|1-q| \gamma(H-U)) d \Gamma} .
$$

If $\gamma>0$, the distribution will be normalizable provided that $\left(\sum p_{i}^{2}\right)^{-\left|\frac{1}{1-q}\right|}$ is integrable over $d^{N D} p$ (for large $p$ ) which is to say that $\left(P^{2}\right)^{-\left|\frac{1}{1-q}\right|} P^{N D-1} d P$ be integrable for $P \rightarrow \infty$; this means we must have $-1>N D-1-2\left|\frac{1}{1-q}\right|$, or $1<q<1+\frac{2}{N D}$ which condition reduces to the classical Boltzmann result $(q=1)$ in the thermodynamic limit 2 .

The other possibility is $\gamma<0$. We then write the distribution as

$$
\rho(\Gamma)=\frac{(1-|\gamma||1-q|(H-U))^{-\left|\frac{1}{1-q}\right|} \Theta(1-|\gamma||1-q|(H-U))}{\int(1-|\gamma||1-q|(H-U))^{-\left|\frac{1}{1-q}\right|} \Theta(1-|\gamma||1-q|(H-U)) d \Gamma}
$$

When integrated over momenta, this expression would show a singularity at $T=\frac{1}{\gamma \gamma|1-q|}+$ $U-V$, unless

$\int\left(X-\sum_{i} p_{i}^{2}\right)^{-\left|\frac{1}{1-q}\right|} d p^{3 N} \sim \int_{0}^{\sqrt{X}}\left(X-P^{2}\right)^{\left|\frac{-1}{1-q}\right|} P^{3 N D-1} d P \sim \int_{0}^{X}(X-Y)^{\left|\frac{-1}{1-q}\right|_{Y} \frac{3 N D-2}{2}} d Y$ is finite; this requires that $1-\left|\frac{1}{1-q}\right|=1-\frac{1}{q-1}>0$, i.e. $q>2$. But $\rho^{q}(\Gamma)$ must also be integrable and in order that the singularity be integrable imposes $1-\frac{q}{q-1}>0$ which is incompatible with the condition $q>2$.

Furthermore the maximization condition demands that the second derivative of the $q$ entropy (1) be $-k_{B} K q \rho^{q-2}<0$, which is satisfied if $q>0$. So in summary, the distribution function exists in the thermodynamic limit for $\gamma>0$ and $0 \leq q \leq 1$, and $\rho(\Gamma)$ has the form of a $q$-exponential with finite support.

Homogeneous entropy. - The homogeneous entropy was proposed as an alternative to the Tsallis entropy because it has various desirable properties that the Tsallis entropy does not share such as being Lesche stable and giving positive-definite heat capacities 13 . It is therefore interesting to ask whether it is subject to the same limitations as found for the Tsallis entropy. The analysis follows essentially the same lines as above except that in this case $\rho(\Gamma)$ is the physical probability 13 and so the energy is computed with the normal average. The homogeneous entropy reads 13

$$
S_{q}^{H}=k_{B} \frac{1-\left(K \int \rho^{\frac{1}{q}}(\Gamma) d \Gamma\right)^{q}}{1-q},
$$

where $K$ is a quantity with the dimensions $[\Gamma]^{\frac{1-q}{q}}$, i.e. $K=\hbar^{D N \frac{1-q}{q}}$, and the normalization and energy constraints are

$$
1=\int \rho(\Gamma) d \Gamma \quad ; \quad U=\int \rho(\Gamma) H d \Gamma .
$$

Following a similar analysis as given in the previous section, the conclusions are that the condition for normalizability is $0 \leq q \leq 1$ and $\gamma>0$ in which case the distribution function reads

\footnotetext{
${ }^{2}$ Note that $\rho^{q}(\Gamma)$ must also be integrable which imposes that $-1>N D-1-\frac{2 q}{q-1}$ or $q<1+\frac{2}{N D-2}$, but this condition is weaker than the constraint $1<q<1+\frac{2}{N D}$.
} 


$$
\rho(\Gamma)=\frac{\left(\exp _{q}(-\gamma(H-U))\right)^{q}}{\int d \Gamma\left(\exp _{q}(-\gamma(H-U))\right)^{q}} .
$$

Here $\gamma=\frac{\bar{\gamma}}{\left(K \int \rho^{\frac{1}{q}}(\Gamma) d \Gamma\right)^{q}}$ where $\bar{\gamma}$ is the Lagrange multiplier used to fix the average energy and $\rho(\Gamma)$ has finite support.

Thermodynamic properties. - Given that the formalism is constructed solely on the basis of the three axioms (11), (2) and (3), the consistent way to define the thermodynamic temperature is through the thermodynamic definition $\partial S / \partial U=1 / T$. Considering $q<1$ and $\gamma>0$, we obtain from the Tsallis entropy (1)

$$
T_{q}^{T}=\frac{1}{k_{B} \gamma}\left(K^{\frac{1}{1-q}} \int \exp _{q}(-\gamma(H-U)) d \Gamma\right)^{q-1},
$$

and from the homogeneous entropy (12)

$$
T_{q}^{H}=\frac{q}{k_{B} \gamma}\left(K^{\frac{q}{q-1}} \int \exp _{q}\left(-\frac{\gamma}{q}(H-U)\right) d \Gamma\right)^{1-q}
$$

With the explicit expressions of $\gamma$ (see (9) and (13)), (15) gives $\bar{\gamma}=1 /\left(k_{B} T_{q}^{H}\right)$, the analog of the classical expression, whereas the equivalent relation for the Tsallis temperature is only obtained in the limit $q \rightarrow 1$.

Correspondingly, the expressions for the specific heat $C_{V}=\left(\frac{\partial U}{\partial T_{q}}\right)$ are given by

$$
C_{V}^{T}=\frac{\beta^{T} / \gamma}{\left[\frac{1}{q}\left(\frac{\beta^{T}}{\gamma}\right)^{4} \frac{1}{K^{2} \int \rho^{2 q-1}(\Gamma)\left(\beta^{T}(H-U)\right)^{2} d \Gamma}-2(1-q)\right]}
$$

with the classical notation $\beta^{T}=1 /\left(k_{B} T_{q}^{T}\right)$, and by

$$
C_{V}^{H}=q^{\frac{1}{q}}\left(\frac{\beta^{H}}{\gamma}\right)^{\frac{1-q}{q}} \frac{k_{B}}{K} \int \rho^{\frac{2 q-1}{q}}(\Gamma)\left(\beta^{H}(H-U)\right)^{2} d \Gamma
$$

where $\beta^{H}=1 /\left(k_{B} T_{q}^{H}\right)$, or

$$
C_{V}^{H}=\frac{1}{k_{B}\left(T_{q}^{H}\right)^{2}} \int d \Gamma \rho(\Gamma)(H-U)^{2} \mathrm{C}_{q}(\Gamma)
$$

with $\mathrm{C}_{q}(\Gamma)=q^{\frac{1}{q}}\left(\frac{\beta^{H}}{\gamma}\right)^{\frac{1-q}{q}} \frac{\rho^{\frac{q-1}{q}}(\Gamma)}{K}$. (18) is the generalization of the classical expression of the specific heat given in terms of the energy fluctuations: $C_{V}=\left\langle(\Delta E)^{2}\right\rangle /\left(k_{B} T^{2}\right)$. The thermodynamic temperatures are both positive while the generalized specific heat is only positive-definite in the case of the homogeneous entropy 3

Concluding comments. - Non-exponential distributions are widely observed in nature. Non-extensive thermodynamics was motivated, in part, as a means of explaining the origin of such distributions which arise naturally as a result of maximizing the generalized entropy with the usual constraints of the normalization of the distribution and of fixed average energy. We have shown that when this procedure is applied to Hamiltonian systems, the resulting distributions only exist for the restricted range of $0<q<1+\mathcal{O}\left(\frac{1}{N}\right)$.

\footnotetext{
${ }^{3}$ It was indeed shown that in the Tsallis formulation the specific heat can be negative [10].
} 
Since the so-called "fat-tailed" distributions correspond to $q>1$, this means that generalized thermodynamics cannot be seen as an explanation of their occurrence for these systems. The problem with larger values of $q$ has to do with the existence of the integrals over momenta due to the unboundedness of the kinetic energy. One way around this would be to redefine the formalism so as to restrict the momenta a priori by making the ansatz $\rho(\Gamma)=\Theta\left(T_{*}-T\right) \rho_{*}(\Gamma)$, for some fixed positive number $T_{*}$, throughout the variational problem and maximizing to determine $\rho_{*}(\Gamma)$. However, this is obviously quite artificial and ad hoc since, for example, one could replace the step function by any function of $T$ that goes to zero sufficiently quickly as $T$ grows. This suggests the more straightforward conclusion that in the case of classical Hamiltonian systems, nonextensive thermodynamics does not provide a simple, natural explanation of distributions with fat tails.

$$
* * *
$$

This work was partly supported by the European Space Agency under contract number ESA AO-2004-070.

\section{REFERENCES}

[1] A. Scott, ed., Encyclopedia of Nonlinear Science (Taylor and Francis, New York, 2005).

[2] C. Tsallis, J. Stat. Phys., 52, 479 (1988).

[3] C. Beck and E.G.D. Cohen, Physica A, 321, 267 (2003).

[4] J. F. Lutsko and J. P. Boon, Phys. Rev. E, 77, 051103 (2008).

[5] H.L. Swinney and C. Tsallis, eds., Anomalous Distributions, Nonlinear Dynamics, and Nonextensivity, Physica D, 193 (2004); J.P. Boon and C. Tsallis, eds., Nonextensive statistical mechanics: new trends, new perspectives, Europhys. News, 36/6, 183-231 (2005); Chap.7 in 6].

[6] C. Tsallis, Introduction to Nonextensive Statistical Mechanics (Springer, New York, 2009), see Bibliography.

[7] R. Luzzi, A.R. Vasconcelos and J. Galvao Ramos, Science, 298, 1171 (2002); M. Nauenberg, Phys. Rev. E, 67, 036114 (2003); D.H. Zanette and M.A. Montemurro, Physics Lett. A, 316 184 (2003); P. Grasberger, Phys. Rev. Lett., 95, 140601 (2005); D.H. Zanette and M.A. Montemurro, Physics Lett. A, 324, 48 (2007).

[8] A. Cho, Science, 297, 1269 (2002); A. Plastino, Science, 300, 250 (2003); V. Latora, A. Rapisarda and A. Robledo, Science, 300, 250 (2003); C. Tsallis, Phys. Rev. E, 69, 038101 (2004); M. Nauenberg, Phys. Rev. E, 69, 038102 (2004); R. Balian and M. Nauenberg, Europhys. News, 37, 9 (2006); F. Bouchet, T. Dauxois and S. Ruffo, Europhys. News, 37, 9 (2006); A. Rapisarda and A. Pluchino, Europhys. News, 37, 10 (2006); R. Luzzi, A.R. Vasconcelos and J. Galvao Ramos, Europhys. News, 37, 11 (2006); see also discussion in Chap.8 of reference 6].

[9] S. Abe, Europhys. Lett., 90, 50004 (2010).

[10] S. Abe, Phys. Lett. A 263424 (1999); 267456 (2000).

[11] J.P. Boon and J.F. Lutsko, Physics Lett. A, 375, 329 (2011).

[12] B. Lesche, J. Stat. Phys. 27419 (1982); S. Abe, Phys. Rev. E 66046134 (2002).

[13] J.F. Lutsko, J.P. Boon and P. Grosfils, Europhys. Lett., 86, 40005 (2009).

[14] C. Beck and F. Schlögl, Thermodynamics of Chaotic Systems, (Cambridge University Press, London, 1993). 